\title{
The effect of suction on ground surface settlement for a tunnel
}

\author{
Bestun Shwan ${ }^{1, *}$ \\ ${ }^{1}$ Koya University, Geotechnical Engineering Department, Faculty of Engineering, Iraq
}

\begin{abstract}
This paper addresses the physical modelling of a tunnel for saturated and unsaturated sand as well as tests in dry condition. A series of tests were conducted for a $50 \mathrm{~mm}$ diameter tunnel buried 25 $\mathrm{mm}$ into a poorly graded fine sand under different normal stresses: $0.78,1.56,2.34$ and $3.31 \mathrm{kPa}$. Suction was controlled by means of the hanging column technique (HCT). Particle image velocimetry (PIV) was also utilised to obtain the vector displacement and compared with the results of the physical modelling. The results of the fully saturated sample showed that the sample experienced a sudden spatial collapse above and around the tunnel's crown. In addition, large displacement on top of the tunnel's crown was also observed for the fully dry case. However, the unsaturated sample did not experience any sudden collapse. This was attributed to the effect of the suction contribution on strength.
\end{abstract}

\section{INTRODUCTION}

Comprehensive research can be found in the literature characterising the behaviour of buried pipes under external loading conditions, [1-3]. However, only a number of studies have been conducted to study the effect of soil moisture variations on the surface settlement of tunnels, [4] and [5].

On the other hand, numerical modelling to predict tunnelling induced ground settlement has been utilised successfully in the literature. Many constitutive material models based on the finite element method have been used to conduct studies on various saturation conditions $[1,6,7]$. These models predominantly require a cumbersome list of parameters which in turn needs advanced soil testing, good selection and understanding of the parameters.

The effect of climate change on the stability of infrastructure such as tunnels and underground pipelines is significant. For example, wetting and drying cycles have a significant influence on the stability of tunnel induced ground surface stability (surface settlement). Many catastrophic accidents such as tunnel induced sinkholes, plastic collapse, and swelling can be bracketed and attributed to the effect of drying and wetting cycles on strength.

This paper reports a series of $1 \mathrm{~g}$ tests of a tunnel buried $25 \mathrm{~mm}$ into a fine sand at various saturation conditions: dry, saturated and unsaturated. The experimental results of the surface settlement and the particle size velocimetry (PIV) results are presented.

\section{MATERIAL AND RIG USED}

A fine sand classified as poorly graded sand (SP) according to the Unified Soil Classification System (USCS) was used in this study. The dry shear strength parameters: $\phi=36.8^{\circ}$ and $c=0 \mathrm{kPa}$ were obtained using a series of direct shear tests. The initial void ratio was 0.84 corresponds to a dry unit weight of $14.11 \mathrm{kN} / \mathrm{m}^{3}$. Further details with regard to the physical properties and particle size distribution curve for the sand used are given by Shwan [8].

A special chamber for conducting the physical modelling, made of glass, previously designed by [8] was utilised in this study. Figure 1 depicts the schematic diagram of the chamber used with the dimensions. A hollow rigid cylindrical pipe with a wall thickness of 2 $\mathrm{mm}$ was used to represent a buried tunnel. The pipe was located $25 \mathrm{~mm}$ below the soil surface and placed at the mid of the chamber. Three dial gauges were placed on the top of a thin base plate (see Fig. 1-previously located on the sample surface) for surface settlement measurement. The base plate was placed to ensure uniform load distribution over the surface area of the sample via four vertical columns (see Fig. 1). The three gauges were named as left, centre and right in the context of this paper. The central gauge was placed at the middle of the rig while the other gauges were 150 $\mathrm{mm}$ from the central gauge. The entire soil surface was subjected to static normal loadings at four stages. The normal load application values were $0.78,1.56,2.34$ and $3.31 \mathrm{kPa}$. The time interval between each loading was 4 minutes. The time interval was established when no further movements of the installed gauges were observed after applying static load.

\section{SAMPLE PREPARATION}

Tests were conducted on dry, saturated and unsaturated samples, therefore, it was necessary to prepare the sample precisely especially due to the large chamber used. To avoid non-homogeneity of the sample and different void ratios, it was necessary to divide the height

* Corresponding author: bestun.shwan@koyauniversity.org 
of the sample equally into five layers (60 $\mathrm{mm}$ each). The amount of the previously calculated dry sand to fill the exact height of each layer was then checked. Otherwise, sample preparation was repeated.

For the dry sample, the oven-dried sand was rained into the chamber at zero distance from the bottom of the rig using the dry pulivation technique. Next, the tunnel was placed carefully, after pouring $275 \mathrm{~mm}$ of soil measured from the bottom of the box, and then the last $25 \mathrm{~mm}$ of soil was poured. The soil surface was gently levelled off by using a T-Shape scraper. After preparing the sample, a camera was then installed in front of the chamber to capture images every 60 seconds for the PIV analysis.

The saturated sample was prepared by first pouring the required amount of water $(11.5 \mathrm{~kg})$ into the chamber to saturate the sand and then an oven-dried sample was poured into the chamber using the water pulivation technique. At the end of the sample preparation, it was ensured that the water level was on the surface of the sample.

The unsaturated sample was prepared using the same procedure for the saturated sample. For this sample, a special filter layer (see Fig. 1) made of $10 \mathrm{~mm}$ of gravel sandwiched between two pieces of porous plastic was used. After the sample was fully saturated, the chamber was then covered by a piece of latex membrane to avoid evaporation. At this stage, the water level on the soil surface coincided with the water level in the burette. The application of the suction was by expelling the required amount of water $(1098 \mathrm{gm})$ from the burette via a valve and then the sample was left for $24 \mathrm{hrs}$ for equalisation purposes. This served as a conservative equilibrium time since $2 \mathrm{hrs}$ monitoring of the water level in the burette after applying suction indicated stable water level. The applied suction for the unsaturated sample was $2.38 \mathrm{kPa}$. The tested sand has an air-entry value of $1.08 \mathrm{kPa}$ and residual suction of $3.6 \mathrm{kPa}$. Afterward, the sample was ready for conducting the test. After the test, three samples at the surface (left, centre and right) were taken for the water content measurements. The average water content was $19.8 \%$.

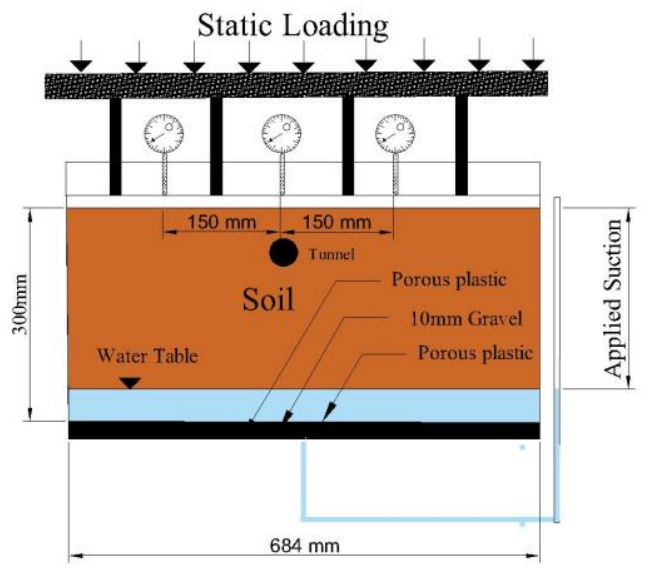

Fig. 1. Schematic diagram of the used chamber.

\section{EXPERIMETAL RESULTS}

Figure 2 depicts the surface settlement versus the normal load for the dry, saturated and unsaturated samples for the middle gauge. For the first three loading stages, the unsaturated sample exhibited higher surface settlement when compared to the dry and saturated samples. The saturated specimen recorded the lowest surface settlement when compared to the unsaturated sample. This behaviour was unexpected and it is under further investigation. However, this may occur due to insufficient loading rate (insufficient time for the excess pore water pressure to dissipate totally when the surface settlement was recorded).

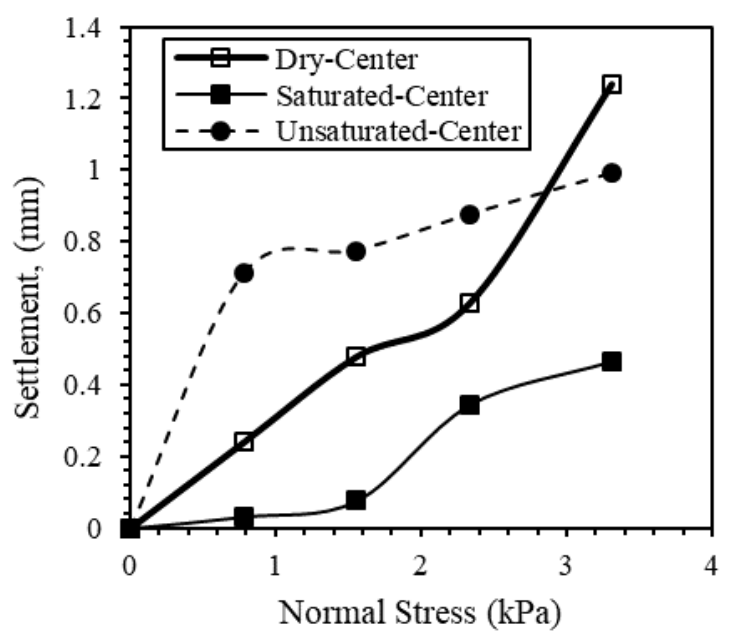

Fig. 2. Surface settlement of the middle gauge for the dry, saturated and unsaturated samples.

Figure 3 represents the transverse settlement trough of the three installed gauges for the four loading stages of the dry, saturated and unsaturated conditions, respectively. The results were fitted with the commonly used empirical equation proposed by [9] which is given by:

$$
S=S_{\max } \cdot e \frac{-x^{2}}{2 i^{2}}
$$

where $S$ is the surface settlement at any point away from the tunnel's centre line by a distance $(x), e$ is exponential constant (2.718), $S_{\max }$ is the maximum central ground settlement at the tunnel centre line and $(i)$ represents the trough width parameter which is the distance from the centre line to the point of inflection of the trough. Equation (1) is based on the Gaussian distribution curve. The total half width of the settlement trough can be assumed to be equal to $2.5 i$ according to [10]. It can be seen, clearly, that the experimental data are reasonably fitted with each loading array (normal distribution curves) obtained using Eq. 1. The maximum ground surface was recorded for the middle guage, except for two readings obtained by the right gauge for the saturated specimen. The findings here are consistent with the common results which can be found in the literature. 


\section{PIV RESULTS}

Figure 2 reveals that the surface settlement values of the unsaturated sample are higher than those for both the fully dry and saturated specimens, except for the last loading stage. Although the comparison showed small change, the contribution of suction on the strength is significant. This required further inspection via the PIV analysis. Table 1 shows the camera setup for the PIV analysis.

Table 1. Setting up the camera for the PIV analysis.

\begin{tabular}{l|l} 
Image sensor & \\
\hline Type & CCD \\
\hline Lens & \\
\hline Focal length & $135 \mathrm{~mm}$ \\
\hline Aperture f/number & $\mathrm{f} / 5.6$ \\
\hline Exposure time & $0.6 \mathrm{sec}$ \\
\hline Recording control & \\
\hline ISO speed equivalent & 100 \\
\hline Flash mode & No flash, compulsory
\end{tabular}

The PIV analysis was performed using the PIVview software,[11], where a pair of images (at the beginning of the test) and after the $4^{\text {th }}$ application of the static loadings were taken. A patch size of $75 \times 34$ was used for all samples. The correlation was based on the most widely used scheme: 3-point Gaussian curve.

Figures $4 \mathrm{a}$ and $\mathrm{b}$ plot the vector displacement results of the PIV analysis for the dry and saturated samples where the expected settlement trough is also drawn according to the results. The PIV results together with captured images showed sudden collapse/large displacements (e.g. see the wild vectors and triangle symbol in Figs. 4a and b) above and around the tunnel for the fully saturated/dry cases, respectively, especially after the application of the $4^{\text {th }}$ loading (e.g. $3.31 \mathrm{kPa}$ ). For a closer inspection of this behaviour, the pair of images before and after the application of the $4^{\text {th }}$ loading was re-analysed using a patch size of $16 \times 8$ for a zone above the tunnel to better capture the behaviour as shown in Figs. $c$ and d.

A sudden collapse above the tunnel for both the dry and fully saturated specimens was obtained (specified as coloured vectors). It is also noticeable that sand particles fell down into the tunnel's portal, especially for the saturated sample. There was a total space of about $1 \mathrm{~mm}$ between the front and back faces of the chamber with the tunnel's face. This is to prevent any friction between the chambers' faces and the tunnel during the loading.

Finally, the PIV results for the unsaturated sample is shown in Figs. 5a and b. The wild vectors (crosses) caused by the effect of the fluorescent tube lambs used in the laboratory. It is intriguing that the sample experienced small internal displacement compared to the dry and fully saturated specimens and with no spatial instability (sudden collapse/large displacements) above and around the tunnel. In addition, there were no signs that particles had fallen down into the tunnel's portal. The results in Figs. 4 and 5 confirm that the fully saturated sample is less stable compared to the dry and unsaturated conditions due to the spatial instabilities. However, such kind of behaviour was not captured by the installed gauges at the surface. On the other hand, the stability of the unsaturated sample is attributed to the increase of the soil strength due to suction contribution.

Distance (mm)
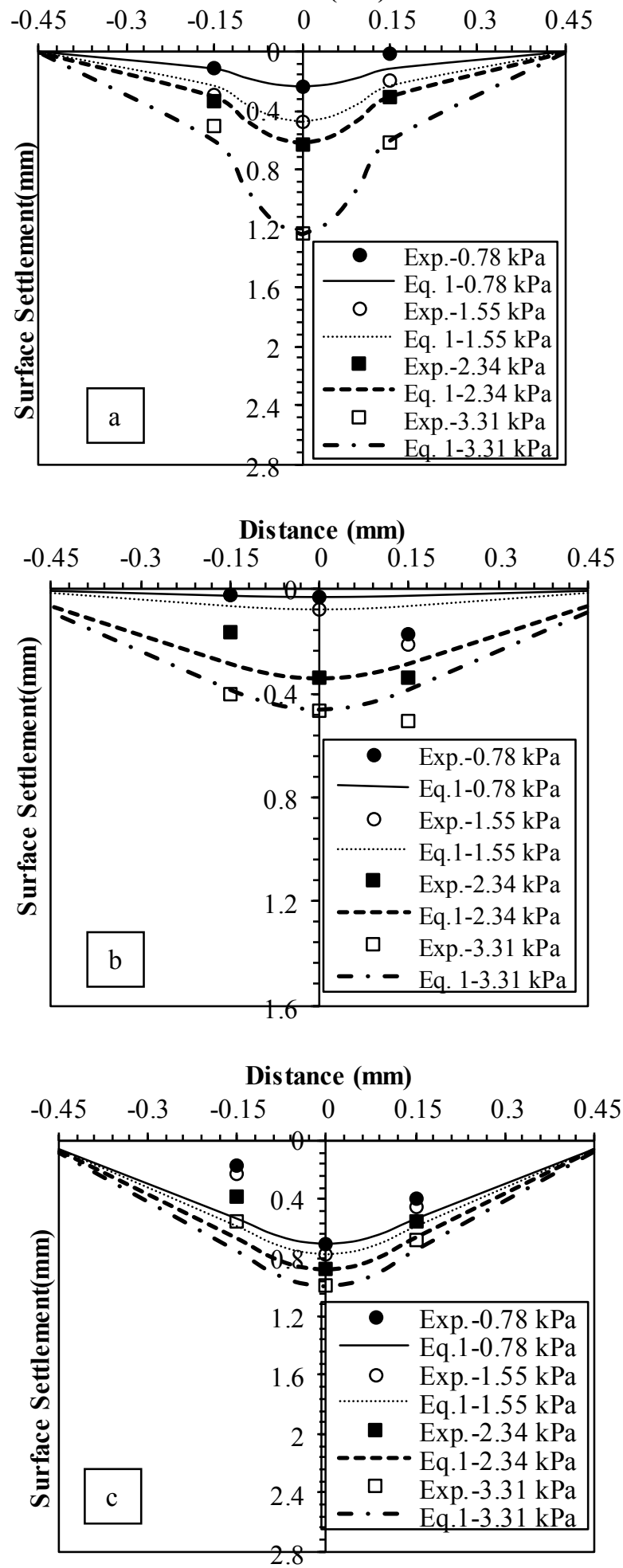

Fig. 3. Experimental and empirical ground settlement trough for the (a) dry, (b) saturated and (c) unsaturated conditions. 


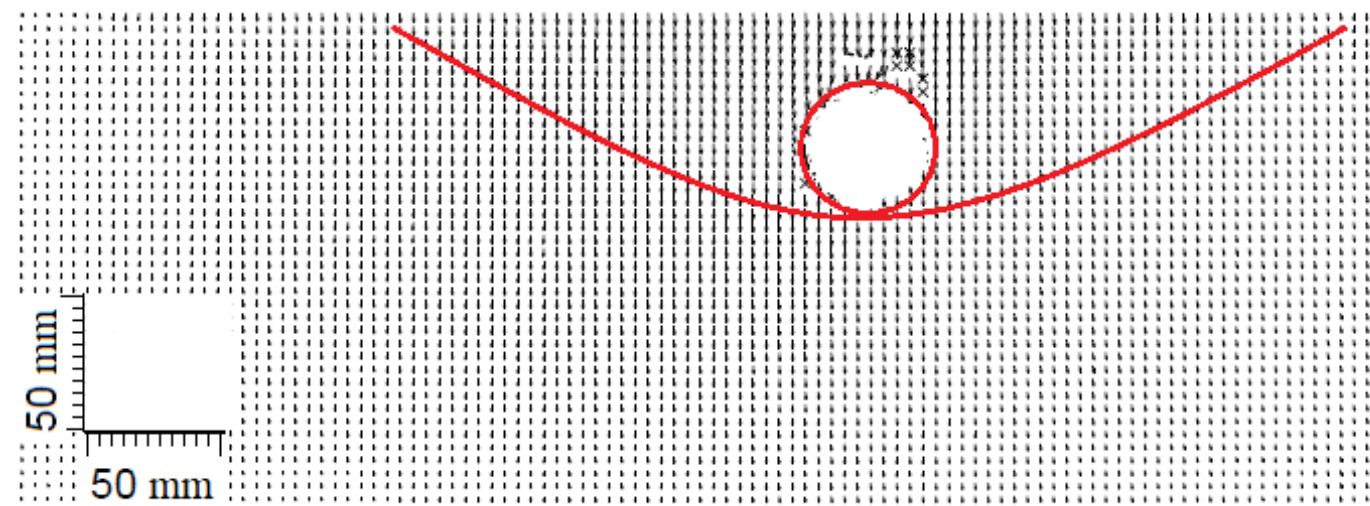

(a)

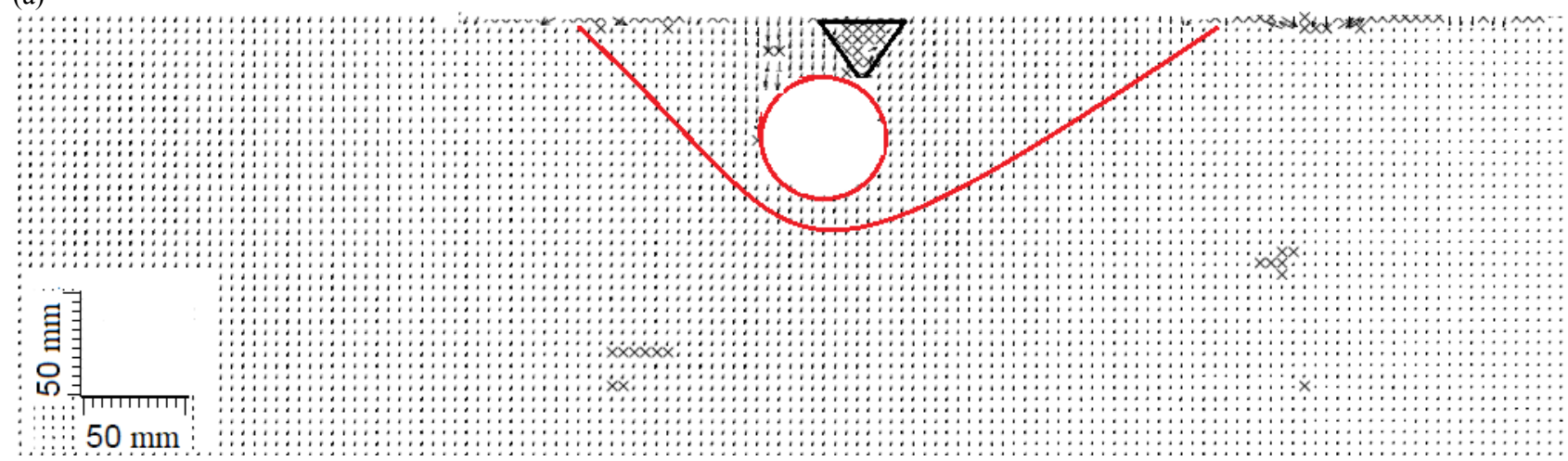

(b)

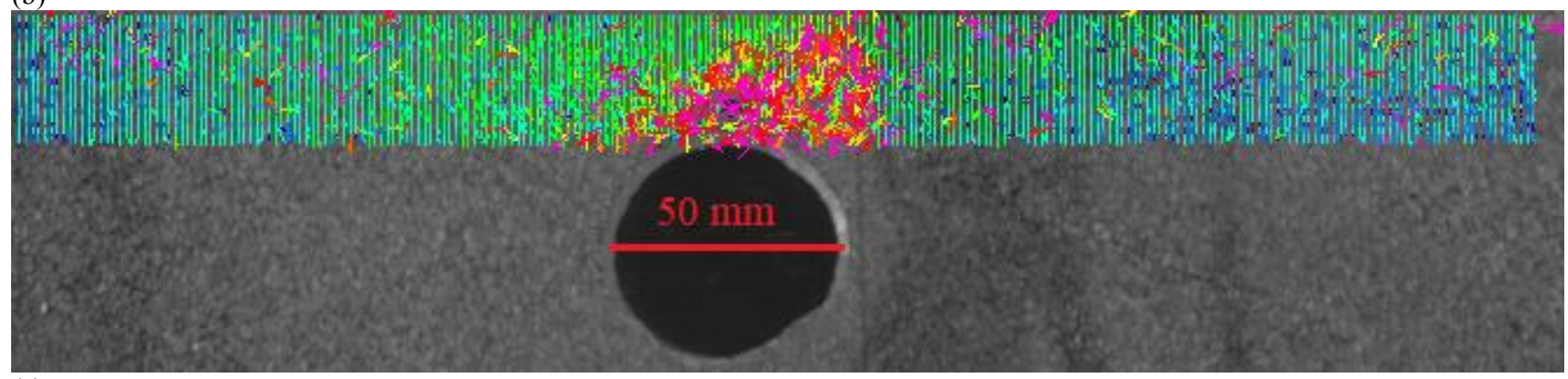

(c)

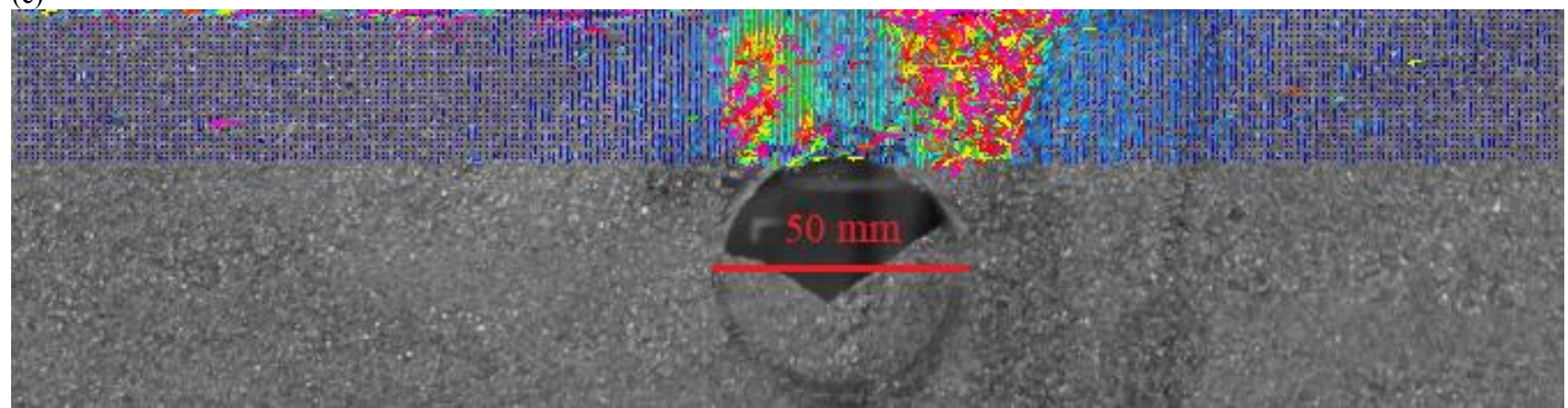

(d)

Fig. 4. PIV results (patch size $=75 \times 34$ ) (a) fully dry case, (b) fully saturated case. PIV results (patch size $=16 \times 8$ ) (c) fully dry case; (d) fully saturated case. 


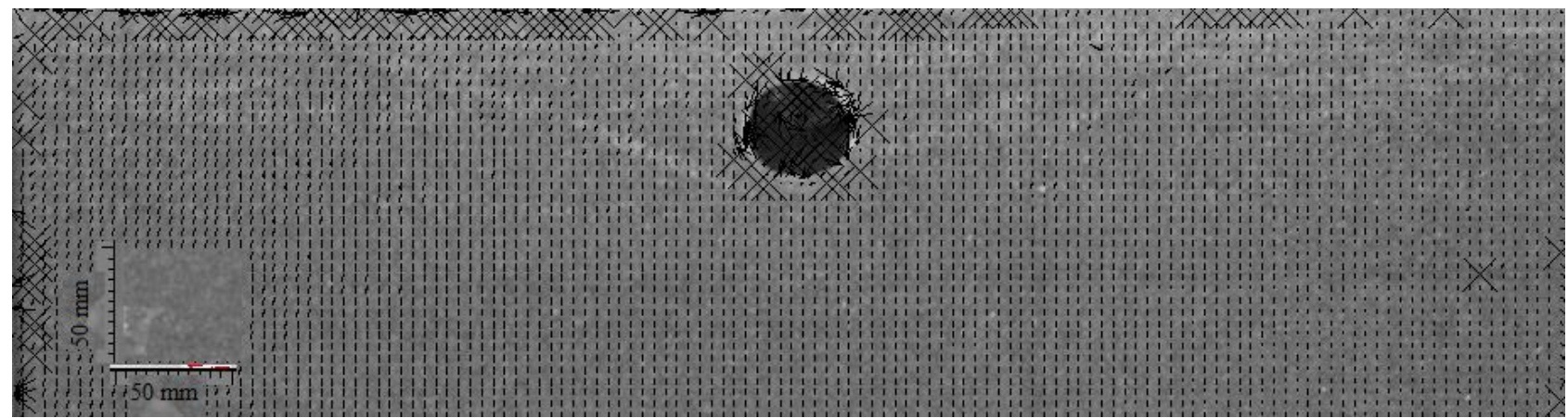

(a)

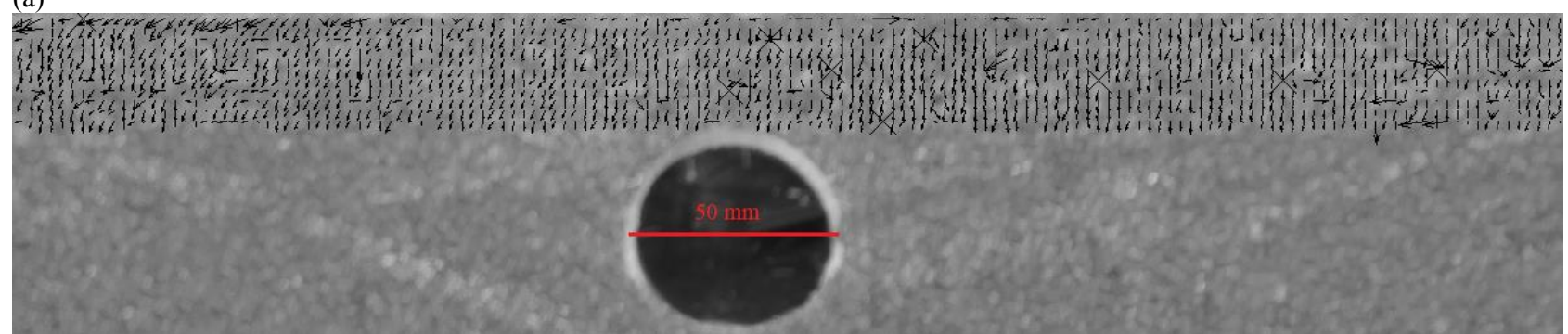

(b)

Fig. 5. PIV results for the unsaturated sample (a) patch size $=75 \times 34$ ) (b) patch size $=16 \times 8$.

\section{CONCLUSIONS}

A series of tests on dry, saturated and unsaturated fine sand samples were conducted using a $1 \mathrm{~g}$ physical modelling of a tunnel buried $25 \mathrm{~mm}$ into the sand. Suction was controlled by means of the hanging column technique (HCT). The tunnel induced transverse settlement trough was obtained based on three installed gauges at the surface. This was fitted using the most commonly used equation proposed by [9] that is based on the normal distribution curve. The analysis was then extended to include particle image velocimetry (PIV). The PIV results showed spatial sudden collapse/large displacement during loading above and around the tunnel's crown for the fully saturated/dry samples, respectively. The PIV results of the unsaturated sample, on the other hand, did not detect any sudden collapse behaviour. The unsaturated sample revealed more stability and less displacement due to the effect of suction which gave rise to the strength.

The author is grateful to Heshu Esmael, Zhala Salah, and Soma Nareeman from the Geotechnical Engineering Department at Koya University for conducting the experimental tests of this paper.

\section{REFERENCES}

1. S. Yimsiri et al., Lateral and upward soil-pipeline interactions in sand for deep embedment conditions. 130(8): p.p. 830-842, (2004).

2. J. Crofts, B. Menzies, and A. J.G. Tarzi, Lateral displacement of shallow buried pipelines due to adjacent deep trench excavations. 27(2): p. 161179, (1977).

3. D. K. Karamitros, et al., Stress analysis of buried steel pipelines at strike-slip fault crossings. 27(3): p. 200-211, (2007).
4. J. K. Jung et al., Lateral soil-pipe interaction in dry and partially saturated sand. 139(12): p.p. 20282036, (2013).

5. D. J. Robert, Soil-pipeline interaction in unsaturated soils. 2010, University of Cambridge.

6. P. Bryden, H. El Naggar, and A.J.G. Valsangkar, Soil-structure interaction of very flexible pipes: centrifuge and numerical investigations. 15(6), (2014).

7. S. Chatterjee, Numerical modelling of pipe-soil interactions. University of Western Australia, (2012).

8. B. J. Shwan, Physical and numerical modelling of sheet pile wall for unsaturated sand. in UNSAT 2018: The 7th Int. Conf. on Unsat. Soils, (2018).

9. R. B. Peck, Deep excavations and tunneling in soft ground. Proc. 7th ICSMFE: p.p: 225-290, (1969).

10. R. J. Mair, R. N. Taylor, and A.J. Bracegirdle, Subsurface settlement profiles above tunnels in clays. Geotechnique. 43(2), (1993).

11. PivView2C, U.M.J.P.G., Germany, Ver. 2.1. 